\title{
The role of high-resolution geomagnetic field models for investigating ionospheric currents at low Earth orbit satellites
}

\author{
Claudia Stolle ${ }^{1,2^{*}}$, Ingo Michaelis ${ }^{1}$ and Jan Rauberg ${ }^{1}$
}

\begin{abstract}
Low Earth orbiting geomagnetic satellite missions, such as the Swarm satellite mission, are the only means to monitor and investigate ionospheric currents on a global scale and to make in situ measurements of $\mathrm{F}$ region currents. High-precision geomagnetic satellite missions are also able to detect ionospheric currents during quiet-time geomagnetic conditions that only have few nanotesla amplitudes in the magnetic field. An efficient method to isolate the ionospheric signals from satellite magnetic field measurements has been the use of residuals between the observations and predictions from empirical geomagnetic models for other geomagnetic sources, such as the core and lithospheric field or signals from the quiet-time magnetospheric currents. This study aims at highlighting the importance of high-resolution magnetic field models that are able to predict the lithospheric field and that consider the quiet-time magnetosphere for reliably isolating signatures from ionospheric currents during geomagnetically quiet times. The effects on the detection of ionospheric currents arising from neglecting the lithospheric and magnetospheric sources are discussed on the example of four Swarm orbits during very quiet times. The respective orbits show a broad range of typical scenarios, such as strong and weak ionospheric signal (during day- and nighttime, respectively) superimposed over strong and weak lithospheric signals. If predictions from the lithosphere or magnetosphere are not properly considered, the amplitude of the ionospheric currents, such as the midlatitude Sq currents or the equatorial electrojet (EEJ), is modulated by 10-15\% in the examples shown. An analysis from several orbits above the African sector, where the lithospheric field is significant, showed that the peak value of the signatures of the EEJ is in error by $5 \%$ in average when lithospheric contributions are not considered, which is in the range of uncertainties of present empirical models of the EEJ.
\end{abstract}

Keywords: Geomagnetic field, lonospheric current, Geomagnetic models

\section{Introduction}

The Earth's magnetic field results from different sources in the Earth's interior, which are moving liquid iron in the outer core, magnetization of the lithosphere and induced currents in the Earth's electrically conductive crust, mantle and ocean, and from electric currents in the ionosphere and magnetosphere. Measurements of the geomagnetic field are an effective and often the only means to characterize these processes and their temporal changes. The separation of the overall observation

\footnotetext{
*Correspondence: claudia.stolle@gfz-potsdam.de

1 GFZ German Research Centre for Geosciences, Potsdam, Germany Full list of author information is available at the end of the article
}

into the contributions from the various sources is a major challenge. This study aims to discuss the separation of the ionospheric signal by predicting other sources with the use of modern geomagnetic models. With the advent of high-precision magnetic field observations onboard scientific satellites like on the Ørsted, CHAMP and Swarm constellation missions, it has been possible to discover and investigate ionospheric currents on a global scale with magnetic signals down to a few nanoteslas. In contrast, ionospheric currents, e.g., during magnetic storms or substorms, such as intense auroral electrojets or fieldaligned currents connecting to the magnetosphere, have magnetic field amplitudes of hundreds or thousands of nanotesla and can easily be identified. Low-amplitude 
currents are always present, independently from the geomagnetic activity level. Ionospheric currents during quiet times constitute a substantial part of the geomagnetic background and are important effects that also have to be dealt with when we want to improve geomagnetic models.

A very efficient approach to studying these currents is to analyze the difference between observations and predictions of effects from non-ionospheric sources derived from geomagnetic field modeling. Obviously, most important is the reduction by the core field, which varies in strength approximately from 20,000 to $70,000 \mathrm{nT}$. A representative empirical model of the core field is the International Geomagnetic Reference Field, IGRF, that provides predictions up to a spherical harmonic degree 13 (Thébault et al. 2015a), corresponding to about 3000$\mathrm{km}$ spatial resolution on ground and low Earth orbit. Additionally, high-resolution empirical models exist that have the capability to predict also the lithospheric field and the quiet-time magnetospheric field dominated by the magnetospheric ring current. These are the CHAOS, GRIMM and POMME model families. Updates have recently been published by Finlay et al. (2015), Lesur et al. (2015) and Maus et al. (2010), respectively. Predictions of the lithospheric signal from these models at satellite altitudes reach typically a couple of nanotesla ( $20 \mathrm{nT}$ or less) in magnitude. The CHAOS-5 model used for this study provides predictions up to a spherical harmonic degree of 100 , corresponding to about $400-\mathrm{km}$ spatial resolution. This paper does not aim at discussing the role of high-resolution lithospheric field models for regional and global geological investigations, but it highlights their relevance for reliably monitoring ionospheric currents at satellite altitudes.

An overlap in magnitude and scale length of the different geomagnetic sources has been demonstrated from global analyses, e.g., in Olsen and Stolle (2012). Here, we want to demonstrate the importance of the application of high-resolution lithospheric and magnetospheric field models for separating and analyzing the ionospheric field. We do this firstly by reviewing recent progress in this field and secondly by highlighting relevant effects with the help of selected orbits of Swarm, representative for magnetically quiet times. To this aim, we want to introduce following notations: Original, in situ observations of the geomagnetic field will in the following be denoted "observations." Results of forward predictions from geomagnetic field models are "predictions," and the difference between observations and predictions [or, in other words, the observations reduced by the model(s)] is named "residuals."

For scientific studies based on residuals between satellite observations and high-resolution geomagnetic field models see, e.g., the review by Olsen and Stolle (2012). Here, we want to provide a short review on publications that are more recent as well as those that have focused on ionospheric currents and that will be relevant later in this study, i.e., currents as the auroral electrojet and fieldaligned currents during geomagnetic quiet times, the dayside mid- and low-latitude Sq currents and the equatorial electrojet. Such quiet-time ionospheric currents show typical spatial and temporal characteristics.

With the aim of characterizing geomagnetic variability in the auroral zones during very quiet times and at low Earth orbit (LEO) altitude, Ritter and Lühr (2006) investigated on the one hand the behavior of residuals in the total field, representing the signals from the auroral electrojet, and on the other hand residuals in the field components perpendicular to the main magnetic field direction, representing signals from field-aligned currents. Here, observations have been reduced by core field predictions and by a homogenous magnetospheric field model based on the Dst index. These authors derived a correlation of 0.5 and higher between perpendicular magnetic deflections (indicating field-aligned currents) and total field residuals (indicating the auroral electrojet) when analyzing both quiet and disturbed conditions and/ or sunlit polar regions, where enhanced conductivity in the E region is expected compared with the dark, quiet regions. Similar good correlations were derived between interplanetary magnetic field proxies and the total field residuals. However, for very quiet conditions and the dark polar hemisphere these correlations are considerably reduced, and variations in the solar wind could not be related to variations in the polar ionospheric currents. During geomagnetic quiet times, the authors also identified stationary anomalies and attributed those to the lithospheric field, which was not accounted when building the magnetic residuals. Recently, based on Swarm constellation residuals to the core, magnetospheric and lithospheric field, Lühr et al. (2015a, b) have derived scale lengths of polar field-aligned currents and found that small-scale currents persist for only about $10 \mathrm{~s}$ at a particular location, while large-scale currents may persist up to $60 \mathrm{~s}$.

A climatology of the dayside, midlatitude Sq current system based on spherical harmonic analysis has been derived from CHAMP data by Pedatella et al. (2011). The authors reduced the observations by the core field and the magnetospheric field and further minimized the effect from radial currents and induction in the solid Earth. In order to achieve a reasonable local time, seasonal and spatial coverage with the satellite data, observations have been collected within \pm 40 days for $1^{\circ} \times 15^{\circ}$ latitude/longitude bins. In comparison with results from a network of ground magnetometers in Europe/Africa, they found 
that amplitude and seasonal behavior of Sq derived from the satellite observations are in good agreement with previous findings from ground stations. The global approach that is possible from satellite data allowed to additionally investigate longitudinal variations of Sq, and they attributed the longitudinal structure to the orientation and strength of the geomagnetic background field and to tidal winds.

A global empirical model for the dayside equatorial electrojet (EEJ) has been derived from the CHAMP mission by Alken and Maus (2007) based on total field residuals to core, lithosphere and magnetosphere models. From this global analysis, they found that the influence of the diurnal eastward-propagating mode with wavenumber-3, DE3, is particularly strong in determining the longitudinal structure of its amplitude, e.g., during equinoxes and June solstice. This feature was also observed for other parameters in the low-latitude upper atmosphere (see for a review, e.g., Oberheide et al. 2015) demonstrating the close relationship between atmospheric tides and ionospheric electrodynamics. Values for EEJ and the eastward electric field are now regularly derived from Swarm observations for each dayside orbit (Alken et al. 2015). From the Swarm constellation, the authors found that the longitudinal gradient for the dayside electric field varies with longitude and amounts up to $0.05 \mathrm{mV} / \mathrm{m} /{ }^{\circ}$ longitude.

High-precision magnetic satellite observations enabled the observation of $\mathrm{F}$ region ionospheric currents at low- and midlatitudes, which are not clearly detectable on ground since they are masked by $\mathrm{E}$ region currents during day or because their signals during nighttime are too weak to be detected at such far distance (see Olsen and Stolle 2012). Recent investigations based on highresolution magnetic field observations from the Swarm constellation mission reduced by the core, lithosphere and magnetospheric field have been used to review fieldaligned and radial currents (Lühr et al. 2015b) as well as diamagnetic and gravity-driven currents associated with typical plasma density structures, at both mid- and low latitudes (Alken 2015).

\section{Data and models}

The Swarm constellation mission was launched on November 22, 2013, into a near-polar, circular orbit of about $500-\mathrm{km}$ altitude. Spacecraft maneuvers started in January 2014 and the three spacecraft reached their final constellation on April 17, 2014. This article will show magnetic field observations from Swarm spacecraft A from the early mission period in December 2013 and January 2014, and the altitude of the spacecraft was about $500 \mathrm{~km}$. The $1 \mathrm{~Hz}$ data of the vector field magnetometer (VFM) are routinely calibrated against the absolute scalar magnetometer (ASM) and are available as Level 1b product (we use version 0405) from http://earth.esa.int/ swarm.

For simplicity, we will in the following refer to differences between observations and predictions from the IGRF (Thébault et al. 2015a) and CHAOS-5 model (Finlay et al. 2015), but our conclusions will be valid for all geomagnetic field models of comparable resolution. Both models are derived from satellite observations from the Ørsted, CHAMP and Swarm missions along with ground-based observatory data. All data are preselected for geomagnetic quiet times and for night (dark) regions to minimize effects of ionospheric and magnetospheric currents leaking into the models. IGRF-12 images the global magnetic field from a set of internal spherical harmonic coefficients up to degree and order 13 , reflecting contributions from the core field. The highresolution model CHAOS- 5 includes different modules to simulate magnetic signatures from different sources. For this study, the core field is predicted from a set of internal spherical harmonic coefficients up to degree 20 and the lithospheric field from degrees from 21 to 100 . For the external field, resulting mainly from the magnetospheric ring current, the spherical harmonic expansion is adopted up to degree 2 and parameterized with the RC index (Olsen et al. 2014), which is a global index of magnetospheric ring current strength derived from several ground observatories.

In the following, we are going to describe the applied types of residuals, and they are summarized in Table 1. Residuals of the magnetic field strengths, $\Delta F$, are obtained from the direct difference: $\Delta F=F_{\text {Meas }}-F_{M}$, where $F_{\text {Meas }}$ are the scalar observations at the ASM instrument on the spacecraft and $F_{M}$ are the predictions derived from a high-resolution magnetic field model, being IGRF and CHAOS-5 here in this study. Furthermore, $F_{M}$ are calculated as the norm of the summed predictions of the magnetic components from a local north-east-center coordinate system $(X, Y, Z)$, so $F_{M}=\sqrt{\left(B_{X, M}\right)^{2}+\left(B_{Y, M}\right)^{2}+\left(B_{Z, M}\right)^{2}}$. To further specify the geomagnetic sources that have been considered in the residuals of $\Delta F_{\mathrm{Io}}$, we introduce the following notations: $\Delta F_{\text {Io }}$ are the residuals to the core, lithospheric and magnetospheric field as described above. Signals from ionospheric sources are still contained in $\Delta F_{\mathrm{Io}}$. $\Delta F_{\mathrm{IoMa}}$ are the residuals to the core and lithospheric field as described above. Residuals $\Delta F_{\mathrm{IoMa}}$ include signals from the ionosphere and the quiet-time magnetosphere. $\Delta F_{\mathrm{IoLi}}$ are the residuals to the core and quiet-time magnetospheric field as described above. Residuals $\Delta F_{\mathrm{IoLi}}$ include signals from the ionosphere and the lithosphere. However, it needs to be mentioned also signatures from induction in the Earth and unmodeled sources leak in 
Table 1 Specification of nomenclature for residuals between observation and model prediction of the magnetic field magnitude. The table names the applied geomagnetic model and the highest degree of spherical harmonics provided by the model for the respective module that has been used to reduce the observations. Colors correspond to applied color lines in Figs. 1, 2, and 3

\begin{tabular}{|c|c|c|c|c|c|}
\hline Nomenclature & $\begin{array}{c}\text { Geomagnetic } \\
\text { model }\end{array}$ & Core & Magnetosphere & Lithosphere & $\begin{array}{c}\text { Included } \\
\text { signals from }\end{array}$ \\
\hline$\Delta \boldsymbol{F}_{\text {Io }}$ & CHAOS-5 & 20 & 2 & 100 & Ionosphere \\
\hline$\Delta \boldsymbol{F}_{\text {IoLi }}$ & CHAOS-5 & 20 & 2 & - & $\begin{array}{c}\text { Ionosphere, } \\
\text { Lithosphere }\end{array}$ \\
\hline$\Delta \boldsymbol{F}_{\text {IoMa }}$ & CHAOS-5 & 20 & - & 100 & \begin{tabular}{c} 
Ionosphere, \\
Magnetosphere \\
\hline$\Delta \boldsymbol{F}_{\text {IGRF }}$
\end{tabular} \\
\hline & IGRF-12 & 13 & - & - & $\begin{array}{c}\text { Ionosphere, } \\
\text { Lithosphere, } \\
\text { Magnetosphere }\end{array}$ \\
\hline
\end{tabular}

all the residuals. We will also use residuals of the components of the geomagnetic field. The residuals in $\mathrm{X}$ direction are labeled $\Delta B_{\mathrm{X}}$, Io and are directly derived as $\Delta B_{X}$, Io $=B_{X \text {, Meas }}-B_{X, M}$. This notation is correspond-

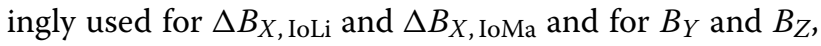
respectively. Residuals to the IGRF model, where the core field is predicted up to degree 13 , are named $\Delta F_{\text {IGRF }}$ and $B_{X, M}=B_{X, \mathrm{IGRF}}$, etc.

\section{Findings and discussion}

In order to exclude storm time effects in the magnetic data, we identified days between December 2013 and December 2014 with the following selection criteria based on geomagnetic indices: Dst $>-20 \mathrm{nT}$ and $\mathrm{AE}<100 \mathrm{nT}$ during $00-24 \mathrm{UT}$ of the respective day and $\mathrm{Kp} \leq 1$ during 00-24 UT of the respective day and of the two preceding days. We also looked for local times for which we either can expect pronounced features of typical ionospheric currents, or periods for which ionospheric currents are very small or absent. Therefore, we concentrated on periods when satellite Swarm A was in a noon-midnight orbit. At noon, the EEJ and the Sq currents are largest and their signatures are clearly expected in the magnetic field. At midnight, E region currents at mid- and low latitudes are absent, and we expect reduced $\mathrm{F}$ region currents, such as diamagnetic and gravitydriven currents, e.g., compared with post-sunset hours where these currents can reach several nanoteslas in the magnetic field at low Earth orbit altitude (e.g., Alken 2015). Between December 2013 and December 2014, we identified orbits from 21 days that meet these criteria. Out of these days, we choose December 12, 2013, for which the Dst index varied between 2 and $-11 \mathrm{nT}$, and the auroral electrojet index (AE index) did never exceed $89 \mathrm{nT}$.

In order to be able to discuss representative examples, we further identified orbits where the lithospheric field is pronounced (i.e., above the African continent) and another orbit where it is weak (i.e., above the Pacific). The two dayside orbits have been selected since those show clear signatures of the Sq currents at the Southern Hemisphere. We believe that this selection is representative for a broad range of typical scenarios.

Figure 1 shows differences of the magnetic field magnitude, $\Delta F$, between observations and IGRF and CHAOS-5 model predictions for the three selected orbits on December 12, 2013, for Swarm spacecraft A, plotted over quasi-dipole (QD) latitude. The QD magnetic coordinate system is described in Emmert et al. (2010). The lower panels show the magnetic local time of the respective orbital segments. The left panel represents a descending orbital part located around local midnight and the middle and right panel ascending orbits around local noon. The black line shows $\Delta F_{\mathrm{Io}}$ time series and includes signatures from ionospheric currents. As expected for midand low latitudes ( $\angle 60^{\circ}$ QD latitude) at the nightside, the 


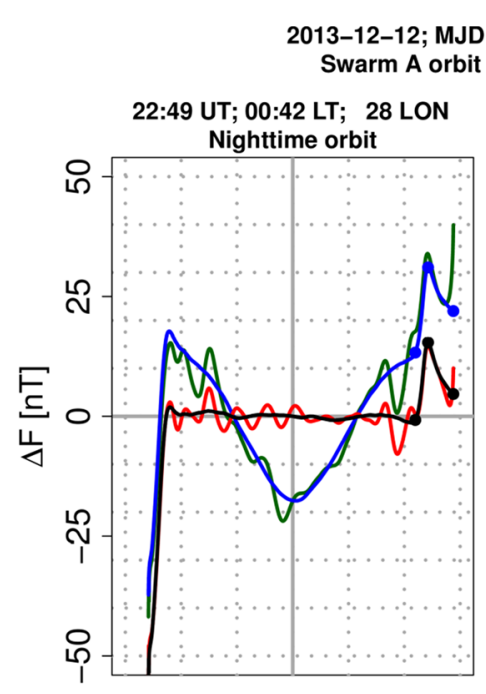

2013-12-12; MJD 5094; DoY: 346

Swarm A orbit no of day 15

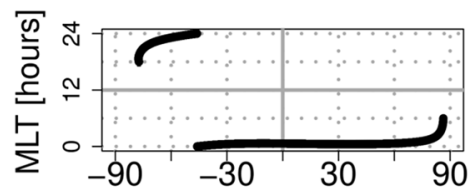

22:03 UT; 12:43 LT; -140 LON Daytime orbit
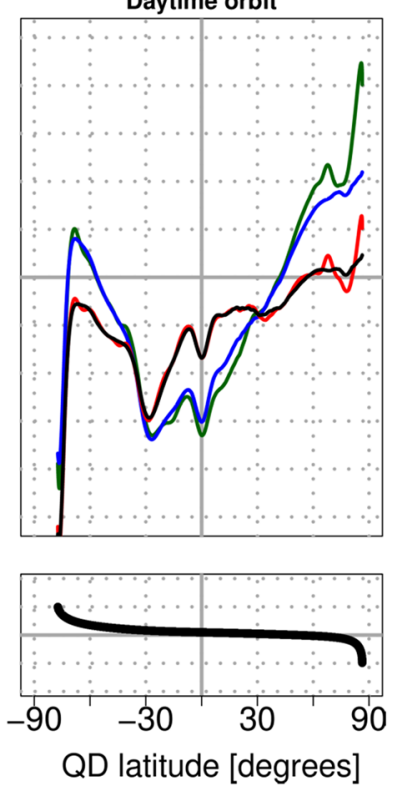

2013-12-12; MJD 5094; DoY: 346

Swarm A orbit no of day 08

11:04 UT; 12:47 LT; 26 LON Daytime orbit
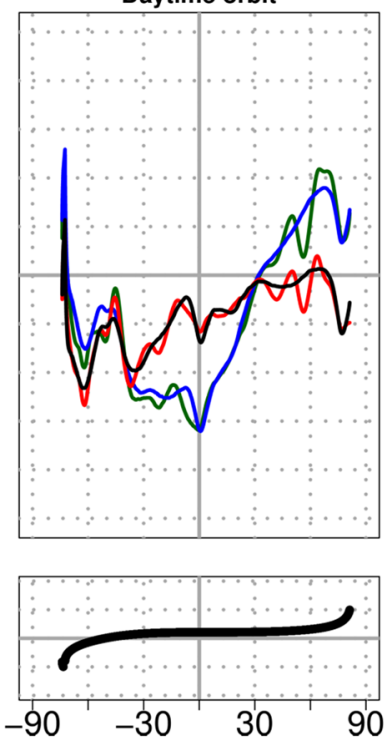

Fig. 1 Time series of $\Delta F$ for three orbital segments of Swarm A. Descending orbit on the nightside (left) and ascending orbit on the dayside (middle and right). Longitude, time and geographic local time when the spacecraft crossed the geographic equator are given on top of the figures. The different colored lines indicate $\Delta F_{10}$ (black), $\Delta F_{\text {loLi }}($ red $), \Delta F_{\text {IOMa }}$ (blue) and $\Delta F_{\text {IGRF }}$ (green). See text for more details. The lower row shows magnetic local time of the respective orbital segment

ionospheric signals are very weak $(<1 \mathrm{nT})$ and can be attributed to remaining gravity-driven currents, plasma pressure gradient-driven currents, or currents produced by the $\mathrm{F}$ region dynamo, or might include other unmodeled sources. For this example orbit, the residual at the magnetic equator vanishes $\left(\Delta F_{\mathrm{Io}}=0 \mathrm{nT}\right)$; thus, the predictions of the magnetospheric field from the CHAOS-5 model did match the observed magnetospheric signature at this time and location very well. The left panel of Fig. 2 shows equatorial segments of all (92) orbits between December 2013 and December 2014 and 23-01 LT for days with $\mathrm{Kp} \leq 1$ and Dst $\geq-20 \mathrm{nT}$. The mean of largescale equatorial $\Delta F_{\text {Io }}$ is about $+2 \mathrm{nT}$ and in a few cases reaches up to $\pm 10 \mathrm{nT}$. This implies that the recent models of the magnetospheric field in certain cases slightly under- or overestimates the magnetospheric effects. One reason could be that the $\mathrm{RC}$ index, although derived from observatories located at different longitudes, provides a global mean value that cannot reflect potential local time and/or longitudinal variations of the magnetospheric ring current. However, we did not investigate this issue in more detail here.

The dark northern auroral latitudes show signatures of some $+20 \mathrm{nT}$ caused by the auroral electrojet, and this is consistent with regular activity even during very quiet geomagnetic times (Ritter and Lühr 2006). Auroral field-aligned currents connecting the ionospheric and magnetospheric current systems strongly affect satellite magnetic data since the spacecraft is directly crossing, or in close proximity to, these currents. However, fieldaligned currents have no signatures in the component along the field line and therefore a negligible effect in $\Delta F$. The sunlit southern auroral region is electrically more conductive and carries $\mathrm{E}$ region ionospheric currents with a signature of several tens of nanotesla in the satellite data. The red curve shows $\Delta F_{\mathrm{IoLi}}$ and thus includes ionospheric and lithospheric signals. Here, the satellite crosses the African continent, which exhibits significant crustal magnetization anomalies. The residuals $\Delta F_{\mathrm{IoLi}}$ have amplitudes up to $\pm 10 \mathrm{nT}$ in mid- and low latitudes. The amplitudes and spatial extensions are comparable to that of the signal from ionospheric sources like the (nighttime) auroral and equatorial electrojets and the midlatitude Sq currents. The blue curve shows $\Delta F_{\mathrm{IoMa}}$ and includes the field of the large-scale magnetospheric ring current plus the ionospheric signal. The magnetospheric signal clearly dominates in amplitude, and its magnetic field in first approximation is globally southward directed at the Earth, thus reducing the magnetic field strengths at low latitudes and increasing it at high 

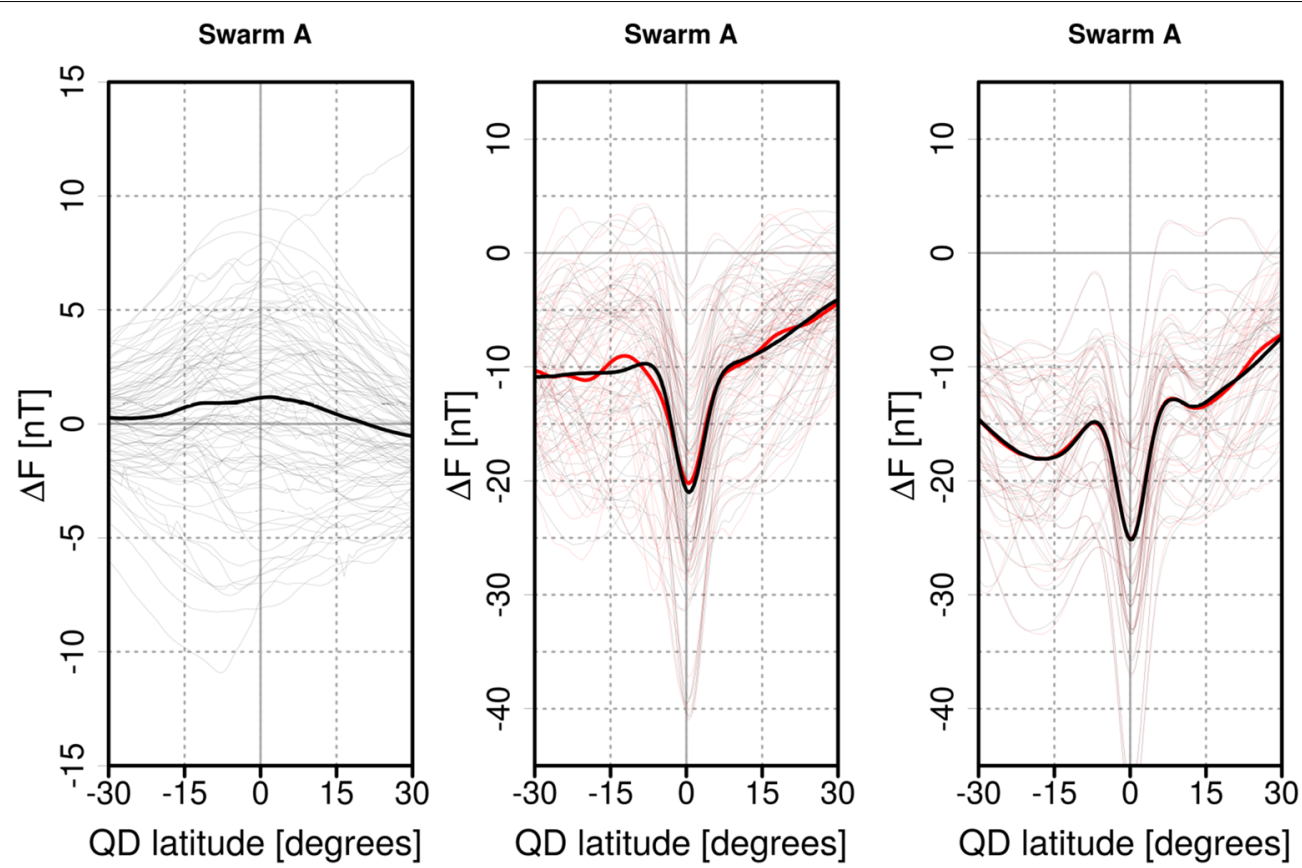

Fig. 2 Thin lines indicate magnetic field residuals along orbits between December 2013 and December 2014 during days with $\mathrm{Kp} \leq 1$ and Dst $\geq-20 \mathrm{nT}$. Thick lines represent the mean of all shown orbits. Left $\Delta F_{10}$ between 23-01 LT. Middle $\Delta F_{10}$ (black), $\Delta F_{\text {loLi }}$ (red), above the African continent between $-10^{\circ}$ and $50^{\circ}$ geographic longitude. Right $\Delta F_{\text {lo }}$ (black), $\Delta F_{\text {loLi }}\left(\right.$ red), above the Pacific Ocean between $-150^{\circ}$ and $-90^{\circ}$ geographic longitude and between 11 and 13 LT

latitudes. The magnetospheric field has its largest impact in leveling magnetic signals from other, e.g., ionospheric sources. As an example, the start and peak points of a magnetic signature caused by an auroral electrojet in the Northern Hemisphere are indicated by thick bullets on the black and blue lines in the left panel of Fig. 1. Taking this example, the difference between the northern polar end of the orbit and the peak in $\Delta F_{\mathrm{Io}}$ at $73^{\circ} \mathrm{N}$ QD latitude is reduced from $10.7 \mathrm{nT}$ in $\Delta F_{\mathrm{IoLi}}$ to $9.1 \mathrm{nT}$ in $\Delta F_{\mathrm{IoMa}}$, while the difference between the equatorward edge of the current signature and the peak in $\Delta F$ at $73^{\circ} \mathrm{N}$ QD latitude are enlarged from 16.2 to $17.8 \mathrm{nT}$, respectively (differences relate to the thick points on the black and blue lines). Both, compression and stretching, amount to a deformation of this ionospheric signal by about $10 \%$ on each side. The large-scale trend that is introduced by missing magnetospheric corrections distorts the apparent shape of ionospheric and lithospheric signals and depends on the strength of the magnetospheric signal that is time varying and has longitudinal dependence (e.g., Newell and Gjerloev 2012). The residuals between the observations and the IGRF predictions, $\Delta F_{\mathrm{IGRF}}$, are shown by the green line. It includes signatures from the magnetosphere and lithosphere. Residuals to IGRF are not suitable to investigate quiet-time currents on the nightside. Without the high-resolution crustal magnetic field model, it would not be possible to identify and interpret the ionospheric signals in the magnetic data except of those from strong electric currents which occur in the sunlit auroral and polar cap region or during substorms.

The middle panel in Fig. 1 shows residuals of a dayside orbit from Swarm A, where E region currents are prominent and dominate the ionospheric magnetic signatures in the low- and midlatitudes. The equatorial electrojet (EEJ) flows along the geomagnetic equator and depresses the magnetic field at satellite altitudes by about $8 \mathrm{nT}$ in this example. Another prominent depression of $\Delta F$ is visible at about $-28^{\circ}$ QD latitude which results from the midlatitude Sq currents that are strong on the local summer hemisphere. This orbit is located over the Pacific Ocean, where the lithospheric magnetization is weak. Its contribution causes only minor modulations of the ionospheric signals at low- and midlatitudes (red and black lines). As mentioned earlier, it is mainly the large-scale magnetospheric field that influences the leveling and thus the shape of other signals. For example, the peak of the southern Sq current signal in the middle panel is shifted from -29.3 to $-33.9 \mathrm{nT}$ and from $-8.2^{\circ}$ QD latitude to $-26.8^{\circ}$ QD latitude (from black to blue curve). At the north polar region, a lithospheric anomaly is present at the same place where auroral currents can be expected with similar amplitudes (compare with the left panel 
of Fig. 1). This orbital segment is located above Alaska, where the crustal magnetization is again enhanced compared with the oceanic crust. Here, the lithospheric model predicts a magnetization anomaly. Reduced by also the lithospheric predictions, only a very weak, if any, ionospheric signal remains in $\Delta F_{\mathrm{IO}}$.

A dayside orbit with significant ionospheric and lithospheric signals is given in the right panel of Fig. 1, showing an orbit above the African continent. Both amplitudes from the EEJ and the Sq currents are significantly disturbed, if predictions of the lithospheric field are not considered. As for the example of the EEJ, the amplitude between $\Delta F_{\mathrm{Io}}$ and $\Delta F_{\mathrm{IoLi}}$ is reduced by $15 \%$ from -13.8 to $-11.6 \mathrm{nT}$. The peak of the Sq current signal is reduced from -19.6 to $-22.8 \mathrm{nT}$ in the southern midlatitudes. The residuals between the observations and the IGRF predictions, $\Delta F_{\mathrm{IGRF}}$, are not suitable to describe properly quiet-time currents in the dayside $E$ region.

Non-storm-related studies using the magnetic field are often concentrated on atmosphere-ionosphere coupling. Investigating the effect of residuals for single orbits is relevant when one is interested in uncertainties of magnetic field residuals during single events. This is the case, e.g., when investigating the effect of stratospheric warming events on the EEJ in satellite data, as such effects are expected to last only a few days (e.g., Park et al. 2012). For climatological investigations based on longer data sets, such as the longitudinal or latitudinal variation of quiet-time ionospheric currents, neglecting the static lithospheric field can add uncertainties in the results, for the example for EEJ studies. The middle panel in Fig. 2 shows results for all (49) orbits between December 2013 and December 2014 over the African continent between $-10^{\circ}$ and $50^{\circ}$ geographic longitude during days with $\mathrm{Kp} \leq 1$ and Dst $\geq-20 \mathrm{nT}$ and between 11 and $13 \mathrm{LT}$, when the EEJ is well developed. The thin black lines show $\Delta F_{\text {Io }}$ for each selected orbit, and the thin red lines show corresponding $\Delta F_{\text {IoLi }}$. The thick lines show the respective means. The seasonal variability and the day-to-day variability of the EEJ are reflected by the large scatter of the profiles. The mean relative difference at the EEJ peak (at the magnetic equator $21-20 \mathrm{nT}$ ) is $5 \%$, and it is often higher for single passes. Alken and Maus (2007) published climatological model of the EEJ for 7 years of magnetic satellite observations from the CHAMP, Ørsted and SAC-C missions. Converting EEJ model estimates at the equator to eastward electric field (considering a conductivity model for conversion), they compared these estimates with electric field observations by the JULIA radar at the Jicamarca observatory located at about $-78^{\circ}$ longitude in Peru. They give an average uncertainty of their model predictions of $55.8 \mu \mathrm{V} / \mathrm{m}$. A typical strength of the dayside equatorial electric field is $0.5 \mathrm{mV} / \mathrm{m}$ (see their Figure 7), which, in this case, corresponds to an uncertainty of about $10 \%$. Alken et al. (2015) compared direct outputs of electric field predictions derived from EEJ observations by CHAMP and Swarm, and similar deviations between the model prediction and JULIA observations of about 5-10\% occur for a typical value of $0.5 \mathrm{mV} / \mathrm{m}$ (their Table 1). The discussed climatological models analyzed magnetic observations with corrected lithospheric field, so the estimated error probably reflects other model uncertainties. However, they are in a similar range as the uncertainties that are introduced through missing lithospheric contributions in regions where the lithospheric field is significant, such as above the African continent. We therefore conclude that correcting for the lithospheric field in satellite magnetic data is significant when climatological models for longitudinal variations of ionospheric currents during quiet times are derived. For comparison, the right panel in Fig. 2 shows results for all (38) orbits between December 2013 and December 2014 over the Pacific ocean between $-150^{\circ}$ and $-90^{\circ}$ geographic longitude during days with $\mathrm{Kp} \leq 1$ and Dst $\geq-20 \mathrm{nT}$ and between 11 and $13 \mathrm{LT}$. The lithospheric signatures are low above the Pacific, and as expected, the ionospheric residuals are almost not affected by them.

The Sq current is a dayside current that is flowing anticlockwise at the Northern Hemisphere and clockwise at the Southern Hemisphere. The current flow is around a focus that is located at midlatitudes approximately around noon but can shift by about \pm 1 LT hour depending on season, longitude or lunar age (e.g., Yamazaki et al. 2011; Stening and Winch 2013). At the focus, the horizontal magnetic signature from the $E$ region current minimizes in amplitude, while the vertical component, $Z$, peaks. Therefore, Sq current circuits are better investigated from the magnetic field components than from the total field. Figure 2 shows $\Delta B$ time series for the $B_{X}, B_{Y}$ and $B_{Z}$ components for the orbit from the middle panel in Fig. 1. In Fig. 3, the Sq signature shows a zero crossing of $\Delta B_{X}$, Io (upper left panel) collocated with the maxi-

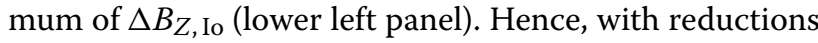
in core, lithosphere and magnetospheric predictions from a high-resolution magnetic field model, the focus of Sq is estimated at $-30^{\circ}$ QD latitude for this example. The identification of the $\mathrm{Sq}$ focus here would not have been possible without the reduction by a quiet-time magnetospheric model, as is shown by the blue and green lines. Note, however, that we did not take into account interhemispheric field-aligned currents that flow between the southern and the northern foci due to electric potential differences, mainly driven by conductivity differences between both hemispheres. These interhemispheric field-aligned currents are expected to contribute a few 

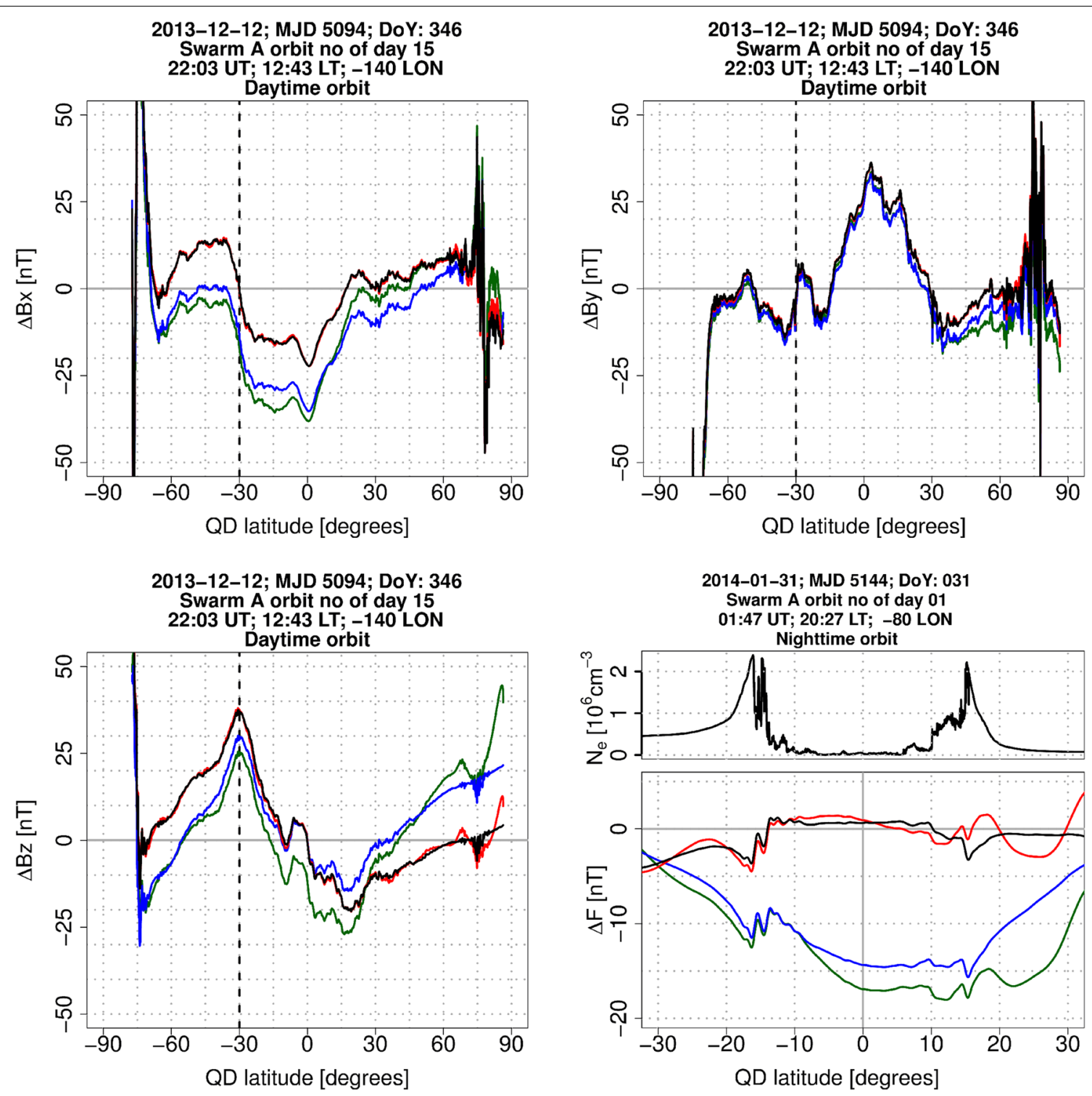

Fig. 3 Time series of $\Delta B_{X}, \Delta B_{Y}$ and $\Delta B_{Z}$ for the daytime orbital segment of Swarm $A$ at 22:03 UT from Fig. 1. The vertical dashed lines indicate the latitude where $\Delta B_{Z}$ has a maximum and $\Delta B_{X}$, Io is $0 \mathrm{nT}$. The lower right panels show electron densities $N_{e}$ and $\Delta F$ for a nighttime orbit on January 31 , 2014. Color codes as for Fig. 1

nanoteslas in the horizontal components at LEO satellites (Pedatella et al. 2011; Park et al. 2011). We also did not take into account induction currents in the crust, mantle and ocean that can have effects of few nanoteslas (e.g., Kuvshinov et al. 2007). Thus, the amplitudes of the determined Sq system may further change when considering all these aspects as well. However, our example demonstrates that neglecting the quiet-time magnetospheric signature predicted by a geomagnetic model can impact the leveling of the zero crossing of the northward component $\Delta B_{X}$ locally by $20 \mathrm{nT}$, even during very quiet times. Also, the magnetospheric ring current reduces the downward magnetic component, $\Delta B_{Z}$, in the Northern Hemisphere and enhances it in the Southern hemisphere since it is southward directed at the Earth. This behav-

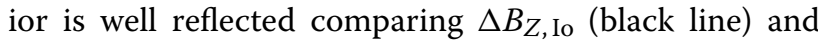
$\Delta B_{Z, \text { IoMa }}$ (blue line) in the lower left panel of Fig. 3 and hence results in a depression of the peak magnitude of the vertical component in the apparent focus of the $\mathrm{Sq}$ in the Southern Hemisphere. Interestingly, $\Delta B_{Z, \text { IGRF }}$ (green line) shows consistently lower values than $\Delta B_{Z}$, IoMa (blue line) between $-50^{\circ} \mathrm{QD}$ and $+50^{\circ} \mathrm{QD}$ latitude. 
Such deviations may result from the short wavelengths (degree > 13) due to the difference in highest spherical harmonic degree of the core field description by IGRF and CHAOS-5, or from a difference in the representation of the secular variation. The evaluation of the different models of the core field is not subject to this study. For more details of model comparison, the reader is referred to Thébault et al. 2015b. Here, we want to reveal that considering quiet-time ring current signatures improves the interpretation of ionospheric signals. The magnetospheric ring current has least effects in the $y$ component at Earth's surface and in low Earth orbit. Therefore, ionospheric signatures are almost not modified by ignoring it, as is visible from the similarities in, $\Delta B_{Y \text {, IoMa (blue line) }}$

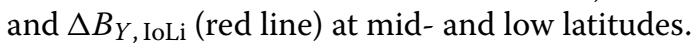

Auroral field-aligned currents have strong magnetic signatures in the components perpendicular to the magnetic field, which is reflected in $\Delta B_{\mathrm{X}}$ and $\Delta B_{Y}$ compared with much lower fluctuations in $\Delta B_{Z}$ being in close direction to the magnetic field lines of high inclination at high latitudes. For the horizontal components reflecting field-aligned currents with several tens of nanotesla, the corrections for the lithospheric and magnetospheric predictions get less important in this example.

The lower right panel in Fig. 3 shows another orbital segment at low latitudes and during local evening hours at 20.27 LT on January 21, 2014, with Dst $\geq-11 \mathrm{nT}, \mathrm{AE} \leq 57$ $\mathrm{nT}$ and $\mathrm{Kp}=0$ throughout the day and $\mathrm{Kp} \leq 2$ on the preceding two days. After sunset, the equatorial ionosphere is regularly affected by equatorial plasma irregularities that may exhibit strong plasma density gradients and create diamagnetic currents with signatures of up to a few nanoteslas in the magnetic field strengths. The scale and amplitude are overlapping with those of the lithospheric field, and their detection is affected when the satellite crosses regions of significant crustal anomalies. Figure 3 shows such an example with diamagnetic currents that are reflected in the magnetic field strength, $F$. The close correlation and the colocation of the magnetic variations with plasma depletions detected in situ by the spacecraft's Langmuir probe confirm their ionospheric origin. On the Southern Hemisphere, the crustal anomalies are very small here and do not influence the detection of ionospheric signals. On the Northern Hemisphere, a crustal anomaly exists at the position of the plasma irregularity. This example shows that neglected lithospheric signals can introduce systematic magnetic signatures in ionospheric studies, e.g., by frequent false detections of ionospheric currents at locations with strong lithospheric magnetic anomalies. On the other hand, the diamagnetic currents can disturb lithospheric modeling. Diamagnetic currents show a typical seasonal and longitudinal distribution, they increase considerably with increasing solar flux, and they are enhanced with proximity to the $\mathrm{F}$ region, where the background electron density is increased (Stolle et al. 2008). These criteria are useful when selecting data for global lithospheric magnetic field modeling from LEO satellite observations.

\section{Conclusions}

High-precision geomagnetic field observations at scientific LEO satellite missions have brought up observational evidence for current systems in the ionospheric $\mathrm{F}$ region such as diamagnetic and gravity-driven, and field-aligned currents in the low- and midlatitudes. High-precision geomagnetic field observations at scientific LEO satellite missions have also successfully been applied for global studies of E region currents, such as Sq currents, the equatorial electrojet and quiet-time polar electrojet and field-aligned currents. These ionospheric currents usually have low amplitudes (a few to few tens of nanotesla in the magnetic field) and exist independently of geomagnetic activity. By using selected examples of Swarm observations, we showed that the investigation of these currents is especially effective when magnetic signatures from other sources, such as from the lithosphere and the quiet-time magnetospheric ring current, are considered and corrected for. In particular, we discussed residuals of the geomagnetic observations to predictions from geomagnetic field models, a method that has successfully been applied in earlier studies on ionospheric currents during quiet magnetic activity. For simplicity, we applied residuals obtained with the IGRF and CHAOS-5 geomagnetic field models, but our conclusions are valid for all models with comparable resolution. At locations of lithospheric magnetic anomalies with signatures of a few nanoteslas at satellite altitudes, the quantitative analysis of ionospheric currents, such as the dayside Sq and EEJ, nighttime plasma pressure-driven currents or quiet-time auroral currents is severely hampered. Signatures from the magnetospheric ring current that are not correctly accounted for can deform amplitudes resulting from ionospheric currents by adding a large-scale, nonlinear trend to the magnetic observations. Both lithospheric and quiet-time magnetospheric signals have been shown to lead to deviations in the estimation of ionospheric signatures in the order of $10-15 \%$ in magnitude in the presented examples. Such modifications are systematic with respect to longitude. An analysis from several orbits above the African sector, where the lithospheric field is significant, showed that the peak value of the signatures of the EEJ is misinterpreted in average by $5 \%$ when lithospheric contributions are not considered, which is in the range of uncertainties of present empirical models of the EEJ providing model uncertainties of about $10 \%$ also when they are based on data that considered the lithospheric signature correction. 
We have demonstrated that the application of geomagnetic field models with a resolution that goes beyond the resolution of the IGRF is a crucial prerequisite for a valid interpretation of quiet-time ionospheric currents detected onboard satellites. By that, scientific geomagnetic satellites provide the only means for the quantification of ionospheric currents on a global scale, e.g., during quiet geomagnetic times.

\section{Authors' contributions}

CS compiled the letter for content and interpretation of the results. IM provided the magnetic field residuals and assisted in providing the figures displayed in this letter. JR produced the predictions of the IGRF and CHAOS-5 models. All authors read and approved the final manuscript.

\section{Author details}

${ }^{1}$ GFZ German Research Centre for Geosciences, Potsdam, Germany. ${ }^{2}$ Faculty of Science, University of Potsdam, Potsdam, Germany.

\section{Acknowledgements}

The authors thank M. Rother and J. Matzka for valuable discussions. The European Space Agency (ESA) is acknowledged for providing Swarm data via http://earth.esa.int/swarm and ftp://swarm-diss.eo.esa.int.

\section{Competing interests}

The authors declare that they have no competing interests.

Received: 27 January 2016 Accepted: 15 June 2016

Published online: 08 July 2016

\section{References}

Alken P (2015) Observations and modeling of the ionospheric gravity and diamagnetic current systems from CHAMP and Swarm measurements. J Geophys Res. doi:10.1002/2015JA022163

Alken P, Maus S (2007) Spatio-temporal characterization of the equatorial electrojet from CHAMP, Ørsted, and SAC-C satellite magnetic measurements. J Geophys Res 112:A09305. doi:10.1029/2007JA012524

Alken P, Maus S, Chulliat A, Vigneron P, Sirol O, Hulot G (2015) Swarm equatorial electric field chain: first results. Geophys Res Lett 42:673-680. doi:10.100 2/2014GL062658

Emmert JT, Richmond AD, Drob DP (2010) A computationally compact representation of magnetic Apex and Quasi-Dipole coordinates with smooth base vectors. J Geophys Res 115:A08322. doi:10.1029/2010JA015326

Finlay CC, Olsen N, Toeffner-Clausen L (2015) DTU candidate field models for IGRF-12 and the CHAOS-5 geomagnetic field model. Earth Planets Space 67:114. doi:10.1186/s40623-015-0274-3

Kuvshinov A, Manoj C, Olsen N, Sabaka T (2007) On induction effects of geomagnetic daily variations from equatorial electrojet and solar quiet sources at low and middle latitudes. J Geophys Res 112:B10102. doi:10.10 29/2007JB004955

Lesur V, Whaler K, Wardinski I (2015) Are geomagnetic data consistent with stably stratified flow at the core-mantle boundary? Geophys J Int 201(2):929-946

Lühr H, Park J, Gjerloev JW, Rauberg J, Michaelis I, Merayo JMG, Brauer P (2015a) Field-aligned currents'scale analysis performed with the Swarm constellation. Geophys Res Lett 42:1-8. doi:10.1002/2014GL062453

Lühr H, Kervalishvili G, Michaelis I, Rauberg J, Ritter P, Park J, Merayo JMG, Brauer $\mathrm{P}$ (2015b) The interhemispheric and $\mathrm{F}$ region dynamo currents revisited with the Swarm constellation. Geophys Res Lett 42:3069-3075. doi:10.1002/2015GL063662
Maus S, Manoj C, Rauberg J, Michaelis I, Lühr H (2010) NOAA/NGDC candidate models for the 11 th generation international geomagnetic reference field and the concurrent release of the 6th generation POMME magnetic model. Earth Planets Space 62(No. 10):729-735. doi:10.5047/ eps.2010.07.006

Newell PT, Gjerloev JW (2012) SuperMAG-based partial ring current indices. J Geophys Res. doi:10.1029/2012JA017586

Oberheide J, Shiokawa K, Gurubaran S, Ward WE, Fujiwara H, Kosch M, Makela JJ, Takahashi H (2015) The geospace response to variable inputs from the lower atmosphere: a review of the progress made by Task Group 4 of CAWSES-II. Prog Earth Planet Sci 2(1):2

Olsen N, Stolle C (2012) Satellite geomagnetism. Annu Rev Earth Planet Sci 40(1):441-465. doi:10.1146/annurev-earth-042711-105540

Olsen N, Lühr H, Finlay CC, Sabaka TJ, Michaelis I, Rauberg J, Tøffner-Clausen L (2014) The CHAOS-4 geomagnetic field model. Geophys J Int 197:815827. doi:10.1093/gji/ggu033

Park J, Lühr H, Min KW (2011) Climatology of the inter-hemispheric fieldaligned current system in the equatorial ionosphere as observed by CHAMP. Ann Geophys 29:573-582. doi:10.5194/angeo-29-573-2011

Park J, Lühr H, Kunze M, Fejer BG, Min KW (2012) Effect of sudden stratospheric warming on lunar tidal modulation of the equatorial electrojet. J Geophys Res 117:A03306. doi:10.1029/2011JA017351

Pedatella NM, Forbes JM, Richmond AD (2011) Seasonal and longitudinal variations of the solar quiet $(\mathrm{Sq})$ current system during solar minimum determined by CHAMP satellite magnetic field observations. J Geophys Res 116:A04317. doi:10.1029/2010JA016289

Ritter P, Lühr H (2006) Search for magnetically quiet CHAMP polar passes and the characteristics of ionospheric currents during the dark season. Ann Geophys 24:2997-3009. doi:10.5194/angeo-24-2997-2006

Stening RJ, Winch DE (2013) The ionospheric Sq current system obtained by spherical harmonic analysis. J Geophys Res Space Phys 118:1288-1297. doi:10.1002/jgra.50194

Stolle C, Lühr H, Fejer BG (2008) Relation between the occurrence rate of ESF and the equatorial vertical plasma drift velocity at sunset derived from global observations. Ann Geophys 26:3979-3988. doi:10.5194/ angeo-26-3979-2008

Thébault E et al (2015a) International geomagnetic reference field: the 12th generation. Earth Planets Space 67:79. doi:10.1 186/s40623-015-0228-9

Thébault E, Finlay CC, Alken P, Beggan CD, Canet E, Chulliat A, Langlais B, Lesur V, Lowes F, Manoj C, Rother M, Schachtschneider R (2015b) Evaluation of candidate geomagnetic field models for IGRF-12. Earth Planets Space 67(1):23. doi:10.1186/s40623-015-0273-4

Yamazaki Y et al (2011) An empirical model of the quiet daily geomagnetic field variation. J Geophys Res 116:A10312. doi:10.1029/2011JA016487

\section{Submit your manuscript to a SpringerOpen ${ }^{\circ}$ journal and benefit from:}

- Convenient online submission

- Rigorous peer review

- Immediate publication on acceptance

- Open access: articles freely available online

- High visibility within the field

- Retaining the copyright to your article

Submit your next manuscript at $\boldsymbol{\nabla}$ springeropen.com 